\title{
Validation of IMU-based gait event detection during curved walking and turning in older adults and Parkinson's Disease patients
}

\author{
Robbin Romijnders ${ }^{1,2^{*}}$ (0), Elke Warmerdam ${ }^{1,2}$, Clint Hansen ${ }^{2}$, Julius Welzel ${ }^{2}$, Gerhard Schmidt ${ }^{1}$ \\ and Walter Maetzler ${ }^{2}$
}

\begin{abstract}
Background: Identification of individual gait events is essential for clinical gait analysis, because it can be used for diagnostic purposes or tracking disease progression in neurological diseases such as Parkinson's disease. Previous research has shown that gait events can be detected from a shank-mounted inertial measurement unit (IMU), however detection performance was often evaluated only from straight-line walking. For use in daily life, the detection performance needs to be evaluated in curved walking and turning as well as in single-task and dual-task conditions.

Methods: Participants (older adults, people with Parkinson's disease, or people who had suffered from a stroke) performed three different walking trials: (1) straight-line walking, (2) slalom walking, (3) Stroop-and-walk trial. An optical motion capture system was used a reference system. Markers were attached to the heel and toe regions of the shoe, and participants wore IMUs on the lateral sides of both shanks. The angular velocity of the shank IMUs was used to detect instances of initial foot contact (IC) and final foot contact (FC), which were compared to reference values obtained from the marker trajectories.

Results: The detection method showed high recall, precision and F1 scores in different populations for both initial contacts and final contacts during straight-line walking (IC: recall $=100 \%$, precision $=100 \%$, F1 score $=100 \%$; FC: recall $=100 \%$, precision $=100 \%$, F1 score $=100 \%$, slalom walking (IC: recall $=100 \%$, precision $\geq 99 \%, F 1$ score $=$ 100\%; FC: recall $=100 \%$, precision $\geq 99 \%$, F1 score $=100 \%$ ), and turning (IC: recall $\geq 85 \%$, precision $\geq 95 \%$, F1 score $\geq$ 91\%; FC: recall $\geq 84 \%$, precision $\geq 95 \%$, F1 score $\geq 89 \%$ ).

Conclusions: Shank-mounted IMUs can be used to detect gait events during straight-line walking, slalom walking and turning. However, more false events were observed during turning and more events were missed during turning. For use in daily life we recommend identifying turning before extracting temporal gait parameters from identified gait events.
\end{abstract}

Keywords: Gait, Gyroscope, Older adults, Parkinson, Step detection, Stroke, Turns, Walking, Wearable sensors

*Correspondence: robr@tf.uni-kiel.de

${ }^{2}$ Neurogeriatrics, Department of Neurology, University Hospital Schleswig-Holstein, Arnold-Heller-Straße 3, Haus D, 24105 Kiel, Germany

Full list of author information is available at the end of the article

\section{Background}

Gait is recognized as a surrogate marker of health, and provides essential clinical insights in neurological disease status $[1,2]$. Traditionally, gait has been assessed by visual observation, which suffers from subjectivity and imprecision [3]. To overcome these limitations, multi-camera optical motion capture (OMC) systems can be used, but these systems are relatively expensive and restricted to original author(s) and the source, provide a link to the Creative Commons licence, and indicate if changes were made. The images or other third party material in this article are included in the article's Creative Commons licence, unless indicated otherwise in a credit line to the material. If material is not included in the article's Creative Commons licence and your intended use is not permitted by statutory regulation or exceeds the permitted use, you will need to obtain permission directly from the copyright holder. To view a copy of this licence, visit http://creativecommons.org/licenses/by/4.0/. The Creative Commons Public Domain Dedication waiver (http://creativecommons.org/publicdomain/zero/1.0/) applies to the data made available in this article, unless otherwise stated in a credit line to the data. 
expertise laboratories [4]. Furthermore, there is increasing evidence that the gait pattern observed in clinical gait assessments does not reflect daily-life gait $[5,6]$. Hence, to get a more complete picture of health status, there is an increasing demand for methods that allow for longterm gait monitoring in ambulatory settings. Inertial measurement units (IMUs) provide a promising alternative to assess gait in an objective, unobtrusive and unconstrained manner $[4,7]$.

The term "gait" refers to "the way of walking" $[8,9]$ and human gait is commonly segmented into repetitive gait cycles. A normal gait cycle begins and ends with initial contact (IC), the instance when the foot strikes the ground [10]. The time interval between two consecutive ICs of the same foot is referred to as the gait cycle time or stride time. The time interval between two successive ICs of the opposite feet is called the step time. If, additionally, the event of final foot contact (FC) is considered, then all phases in the gait cycle can be described: swing and stance phase, or single and double support phase [1, 10]. Identification of gait events (GEs) and phases is considered essential for clinical gait assessment [8]. GEs can be detected from a single low back-mounted IMU [1116], however findings suggest that detecting GEs is easier from shank- or foot-mounted IMUs [17-19] where footmounted IMUs increase errors, especially in pathological gait patterns $[19,20]$.

The performance of IMU-based GE detection is, however, often tested only with treadmill walking $[12,14]$ or from walking trials where only the straight-line segments of walking trajectories were included in the analysis [13, $17,21]$. For more complex walking tasks, such as slalom walking or dual-task walking, one often relies on visually counting of the number of steps, which does not allow to assess the time error of the GE detection and is more prone to errors. Whether IMU-based GE detection is still valid in more complex walking tasks is yet to be shown. Daily-life gait is likely influenced by obstacle negotiation (approximately $30 \%$ of daily-life gait is spent along curved trajectories [22, 23]) and dual-/multi-tasking [5].

The aim of this study is therefore to quantify the performance of IC and FC detection in straight-line walking under single-task and dual-task conditions, and to quantify detection performance in curved walking and turning in (healthy) older adults (OA), people diagnosed with Parkinson's disease (PD), and people who have suffered from a stroke (ST).

\section{Methods}

A step was considered as the interval between consecutive ICs of the ipsi- and contralateral foot [10], and corresponding to forward displacement of the foot together with a forward displacement of the trunk [24]. A stride was the interval between two consecutive ICs of the same foot, and as such it was equivalent to the gait cycle and every stride consisted of two steps $[8,10]$.

\section{Study population}

Three different groups were distinguished: (1) OAs with no signs of any movement disorders, (2) PD participants in the medication $\mathrm{ON}$ state, and (3) ST participants (Table 1). For the OAs the minimum age was 60 years. All participants needed to be able to walk independently with or without walking aids. Exclusion criteria were a high fall risk (i.e. $>2$ falls in the last month, as reported by the participant), any impairment that refrained the participant from giving consent to participate in the study, and a score below 20 for the Montreal Cognitive Assessment (MoCA) [25]. All participants gave written informed consent and the study was approved by the ethical committee of the medical faculty at University Hospital Schleswig-Holstein (UKSH), No: D438/18.

\section{Study protocol}

Participants walked a 5-meter distance that was marked at the start and end with two cones, approximately 1 meter apart (Fig. 1). Participants were asked to start walking approximately two steps before the start, and to stop walking approximately two steps after the end. For the analysis of GE detection, we only considered the events that were registered within the 5-meter distance.

The following walking trials were performed:

- straight-line trial, 5 meter, at preferred speed,

Table 1 Demographic data of the study participants summarized by group

\begin{tabular}{lllllll}
\hline Group & Participants (female) & Age (years) & Height $(\mathbf{m})$ & Mass (kg) & $\begin{array}{c}\text { UPDRS-III } \\
\text { Disease } \\
\text { duration } \\
\text { (years) }\end{array}$ \\
\hline OA & $11(2)$ & $71 \pm 9$ & $1.76 \pm 0.07$ & $78.5 \pm 13.5$ & $4 \pm 3$ & $9 \pm 5$ \\
PD & $14(5)$ & $64 \pm 10$ & $1.78 \pm 0.08$ & $91.3 \pm 14.7$ & $29 \pm 21$ & $2 \pm 4$ \\
ST & $9(2)$ & $68 \pm 10$ & $1.75 \pm 0.08$ & $81.3 \pm 18.0$ & $6 \pm 9$ & 2 \\
\hline
\end{tabular}

${ }^{1}$ Unified Parkinson's Disease Rating Scale, part III: Motor Examination, ${ }^{2}$ for PD: the time since first diagnosis, for ST: the time since stroke 


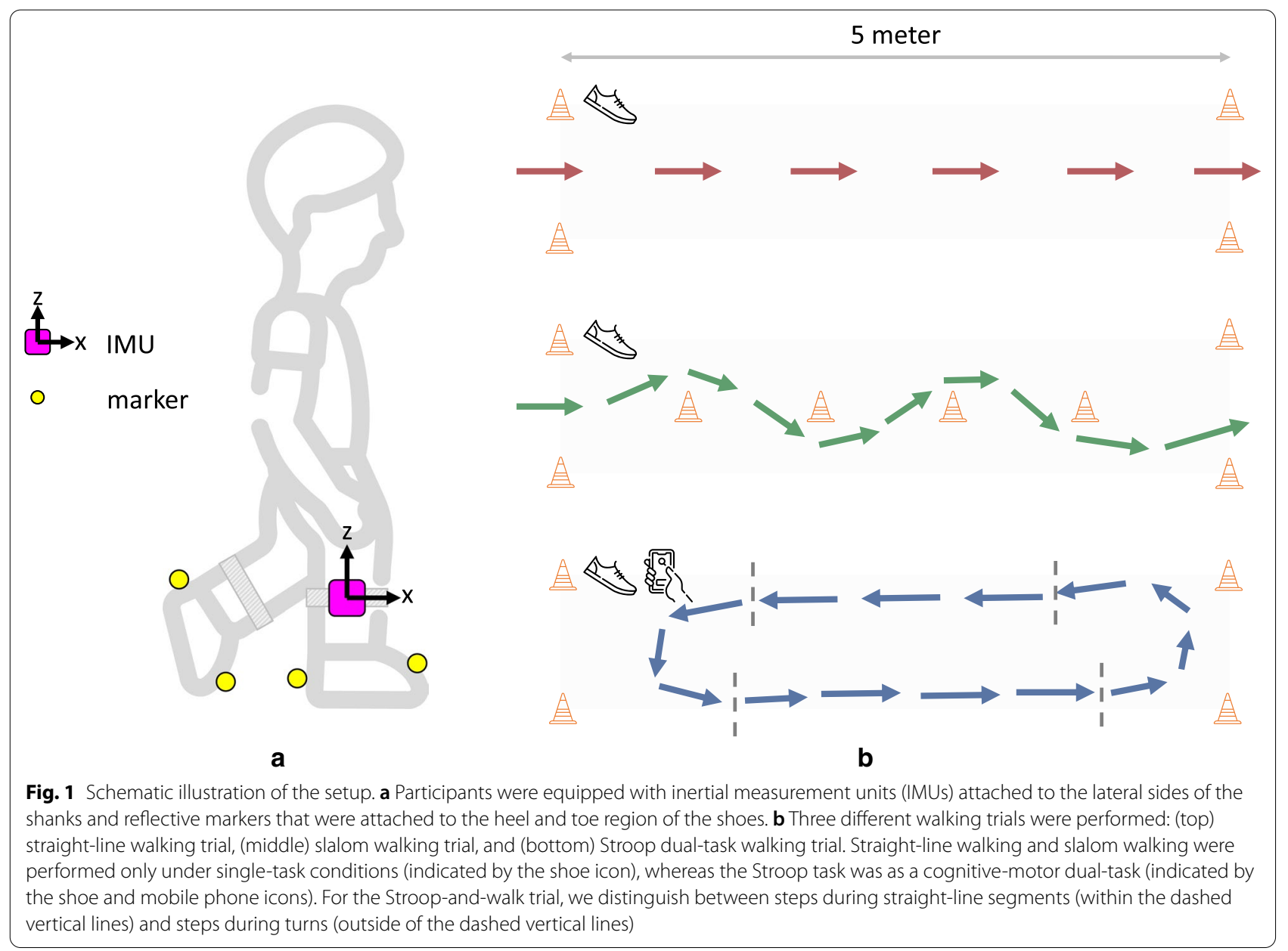

- slalom trial, 5 meter with a cone at every 1 meter, at preferred speed,

- Stroop-and-walk trial, walking up and down the 5-meter distance, while performing a numerical Stroop test [26] on a hand-held mobile phone until completion of the Stroop test, at preferred speed. For the numerical Stroop test, two numbers were displayed on the mobile screen that were different in value and different in semantic size. The participant needed to tap the number with the highest value. No further instructions as to prioritize any task were given.

For the Stroop-and-walk trial, participants started within the 5-meter distance, and walked up and down whilst turning on either end of the 5-meter distance (see Fig. 1 for an example trajectory). Turns were annotated manually using the Qualisys Track Manager 2018.1 software (QTM; Qualisys AB, Göteborg, Sweden), and GEs during turns were analyzed separately.

\section{Optical motion capture system Equipment}

Reflective markers (diameter: $19 \mathrm{~mm}$ ) were attached to the heel and toe of both left and right shoes (Fig. 1). Marker trajectories were recorded by a 12-camera optical motion capture system (Qualysis AB, Götebörg, Sweden) sampling at $200 \mathrm{~Hz}$.

\section{Signal processing}

GEs were detected from the heel marker trajectories, and provided reference values for the GE timings of ICs and FCs. Event timings were based on specific signal features from the heel marker vertical velocity and acceleration, respectively. Raw marker data were loaded into MATLAB (MATLAB 2018b, The Mathworks, Natwick, USA). The raw marker data were first interpolated to fill any gaps [27] and subsequently low-pass filtered with a 4th order Butterworth filter with a cut-off frequency, $f_{\text {cut }}$, of $5 \mathrm{~Hz}$. The filter was applied to the marker data by using MATLAB's built-in filtfilt function, such that the filtered signal was not delayed. 
The timings of ICs correlated with timings of local maxima in the vertical acceleration [28], whereas the timings of FCs closely correlated with timings of local maxima in the heel marker vertical velocity [29]. Like in [30], GEs were checked manually using QTM.

\section{Inertial measurement units \\ Equipment}

One IMU (Noraxon U.S.A. Inc., Scottsdale, Arizona, USA) was attached to each shank with elastic straps. The sampling frequency was also set to $200 \mathrm{~Hz}$ and the OMC and IMUs were synchronized using a trigger at the beginning of each measurement [31].

\section{Signal processing}

Sensor data were loaded into MATLAB. IMUs were aligned such that the sensitive axes pointed roughly in antero-posterior direction (forward being positive), mediolateral direction (left being positive), and the vertical direction (up being positive). Angular velocity was high-pass filtered using an IIR filter with $f_{\text {cut }} \approx 0.15 \mathrm{~Hz}$ to reduce the effect of drift [17], and then low-pass filtered using a 4th order Butterworth filter with $f_{\text {cut }}=10 \mathrm{~Hz}$. Both filters were applied using the filtfilt function.

Detection of GEs using shank-mounted IMUs was based on identifying negative peaks in the medio-lateral angular velocity that was high- and low-pass filtered. These negative peaks closely correlated to timings of mid-swing [17, 18 , 32]. Only negative peaks with a value $\leq 10 \%$ of the global minimum angular velocity were considered. Furthermore, if two or more consecutive peaks were detected within a time interval of $300 \mathrm{~ms}$ of each other, then only the peak with the lowest value was preserved $[21,33]$.

Data from the two legs were analyzed independently of one another to facilitate a setup with an IMU on a single side.

\section{Data analysis}

Two GEs, IC and FC, were extracted using the reference system as well as the shank-mounted IMUs. For both events we evaluated the detection performance in terms of correctly identified events (true positives, $T P$ ), falsely identified events (false positives, $F P$ ) and missed events (false negatives, FN). TPs were defined as $<300 \mathrm{~ms}$ difference (in terms of magnitude) between an event detected by the IMU-based algorithm and the reference event [12]. From these metrics the recall, precision and F1 score were derived:

$$
\text { Recall }=R=\frac{T P}{T P+F N}
$$

$$
\begin{aligned}
& \text { Precision }=P=\frac{T P}{T P+F P} \\
& \text { F1 score }=F 1=2 \frac{P \cdot R}{P+R}
\end{aligned}
$$

Recall expressed how many of the gait events were detected and precision expressed how many of the detected gait events were true gait events. The F1 score can be considered as a weighted average of the recall and precision. Furthermore, algorithm performance was evaluated by assessing the time error between the reference event (from the marker-based algorithm) and the predicted event [34, 35], defined as:

$$
\begin{aligned}
& \epsilon_{\mathrm{IC}}=t_{\mathrm{IC}}-t_{\mathrm{IC}}^{\prime} \\
& \epsilon_{\mathrm{FC}}=t_{\mathrm{FC}}-t_{\mathrm{FC}}^{\prime}
\end{aligned}
$$

where $t_{\mathrm{IC}}$ and $t_{\mathrm{FC}}$ denoted the time of the predicted IC and FC from the IMU-based algorithm, and $t_{\mathrm{IC}}^{\prime}$ and $t_{\mathrm{FC}}^{\prime}$ denoted the reference time of the IC and FC obtained from the OMC.

The effect of dual-task conditions was investigated by comparing GE detection from the straight-line trial to the GE detection from the straight-line segment of the Stroop-and-walk trial (Fig. 1). The effect of curved walking was investigated by comparing GE detection from the straight-line walking to slalom walking and turns from the Stroop-and-walk trial.

\section{Statistical analysis \\ Detection of gait events}

The algorithm performance in detection of GEs was evaluated by generating contingency tables and comparing the recall, precision and F1 scores.

\section{Time agreement}

Time agreement was assessed by determining the mean time error, corresponding 95\% confidence intervals and the mean absolute error (MAE). Confidence intervals (CIs) were computed as $\bar{x} \pm 1.96 s$ with $\bar{x}$ the mean time error, and $s$ the standard deviation of the time errors.

Comparison of time errors between tasks for each group. A Wilcoxon signed-rank test [36] was used to compare each subject's mean values of the absolute errors for the single-task versus the dual-task conditions, and similarly for the straight-line walking, slalom walking, and turns [18]. Differences were considered statistically significant if the p-value was less than 0.05 .

Comparison of time errors between groups for each task. A Wilcoxon rank sum test was used to compare the subject mean values of the absolute errors from the OA 
group and those obtained for the PD and ST group [18]. Differences were considered statistically significant if the p-value was less than 0.05 .

\section{Results}

\section{Detection of gait events}

\section{Effect of dual-tasking}

IMU-based GE detection showed high recall (IC: $\geq 97 \%$, FC: $\geq 96 \%$ ), high precision (IC: $\geq 100 \%$, IC: $\geq 100 \%$ ) and high F1 score (IC: $\geq 99 \%$, FC: $\geq 98 \%$ ) for the three different groups in both single-task and dual-task conditions for GEs during straight walking (Table 2). All ICs and FCs were detected for the single-task trials. In the straight walking segments from the Stroop-and-walk trials a number of false events ( 1 IC, 5 FC) and a number of missed events (18 IC, 28 FC) were observed.

\section{Effect of curved walking and turns}

IMU-based GE detection showed high recall (IC: 100\%, FC: $100 \%$ ), high precision (IC: $\geq 99 \%$, FC: $\geq 99 \%$ ) and high F1 score (IC: $100 \%$, FC: 100\%) for the three different groups for the straight-line walking and slalom walking (Table 3). One IC and one FC were falsely detected for a single PD patient in the slalom walking trial, where the patient swung its foot forward and backward without taking a step (that is, there was a swing phase but the patient did not move forward but rather put its foot down on the same spot). For ICs and FCs during turns, for the three groups, recall was lower than for the straigtline and slalom walking (IC: $\geq 85 \%$, FC: $\geq 84 \%$ ), and likewise for the precision (IC: $\geq 95 \%, \mathrm{FC}: \geq 95 \%$ ) and F1 score (IC: $\geq 91 \%, F C: \geq 89 \%)$. More events were missed by the IMU-based gait event detection (137 ICs, 136 FCs) and more false events were detected (44 ICs, $42 \mathrm{FCs}$ ).

Table 2 Validation results of gait event detection for the straight-line segments of the single-task trial and the Stroopand-walk trial

\begin{tabular}{|c|c|c|c|c|c|c|c|c|c|c|c|c|c|}
\hline & \multirow{3}{*}{$n$} & \multicolumn{6}{|c|}{ Initial contacts } & \multicolumn{6}{|c|}{ Final contacts } \\
\hline & & TP & $\mathrm{FN}$ & FP & $\mathbf{R}$ & $\mathrm{P}$ & $\mathrm{F} 1$ & $\mathrm{TP}$ & $\mathrm{FN}$ & FP & $\mathbf{R}$ & $P$ & F1 \\
\hline & & & & & (\%) & (\%) & (\%) & & & & (\%) & (\%) & (\%) \\
\hline \multicolumn{14}{|c|}{ Straight-line trial } \\
\hline OA & 11 & 83 & 0 & 0 & 100 & 100 & 100 & 83 & 0 & 0 & 100 & 100 & 100 \\
\hline PD & 14 & 131 & 0 & 0 & 100 & 100 & 100 & 133 & 0 & 0 & 100 & 100 & 100 \\
\hline ST & 9 & 78 & 0 & 0 & 100 & 100 & 100 & 81 & 0 & 0 & 100 & 100 & 100 \\
\hline \multicolumn{14}{|c|}{ Stroop-and-walk trial (straight-line segments) } \\
\hline $\mathrm{OA}$ & 11 & 501 & 5 & 0 & 99 & 100 & 100 & 497 & 8 & 1 & 98 & 100 & 99 \\
\hline PD & 11 & 587 & 1 & 1 & 100 & 100 & 100 & 589 & 2 & 2 & 100 & 100 & 100 \\
\hline ST & 9 & 457 & 12 & 0 & 97 & 100 & 99 & 451 & 18 & 2 & 96 & 100 & 98 \\
\hline
\end{tabular}

Table 3 Validation results of gait event detection during straight-line walking, curved walking, and turns

\begin{tabular}{|c|c|c|c|c|c|c|c|c|c|c|c|c|c|}
\hline & \multirow{3}{*}{$n$} & \multicolumn{6}{|c|}{ Initial contacts } & \multicolumn{6}{|c|}{ Final contacts } \\
\hline & & TP & $\mathrm{FN}$ & FP & $\mathbf{R}$ & $P$ & $\mathrm{~F} 1$ & TP & $\mathrm{FN}$ & FP & $\mathbf{R}$ & $P$ & F1 \\
\hline & & & & & (\%) & (\%) & (\%) & & & & (\%) & (\%) & (\%) \\
\hline \multicolumn{14}{|c|}{ Straight-line trial } \\
\hline $\mathrm{OA}$ & 11 & 83 & 0 & 0 & 100 & 100 & 100 & 83 & 0 & 0 & 100 & 100 & 100 \\
\hline PD & 14 & 131 & 0 & 0 & 100 & 100 & 100 & 133 & 0 & 0 & 100 & 100 & 100 \\
\hline ST & 9 & 78 & 0 & 0 & 100 & 100 & 100 & 81 & 0 & 0 & 100 & 100 & 100 \\
\hline \multicolumn{14}{|c|}{ Slalom trial } \\
\hline $\mathrm{OA}$ & 11 & 103 & 0 & 0 & 100 & 100 & 100 & 96 & 0 & 0 & 100 & 100 & 100 \\
\hline PD & 14 & 181 & 0 & 1 & 100 & 99 & 100 & 190 & 0 & 1 & 100 & 99 & 100 \\
\hline ST & 9 & 124 & 0 & 0 & 100 & 100 & 100 & 126 & 0 & 0 & 100 & 100 & 100 \\
\hline \multicolumn{14}{|c|}{ Stroop-and-walk trial (turns) } \\
\hline $\mathrm{OA}$ & 11 & 297 & 51 & 11 & 85 & 97 & 91 & 296 & 55 & 15 & 84 & 95 & 89 \\
\hline PD & 11 & 312 & 45 & 18 & 87 & 95 & 91 & 315 & 42 & 14 & 88 & 96 & 92 \\
\hline ST & 9 & 281 & 41 & 15 & 87 & 95 & 91 & 282 & 39 & 13 & 88 & 96 & 92 \\
\hline
\end{tabular}




\section{Time agreement}

\section{Effect of walking task on gait event detection}

Effect of dual-task walking. Table 4 shows the mean time errors, mean absolute error (MAE) and the 95\% CI for the GEs during straight-line walking, either from the straight-line trial (single-task) or the Stroop-and-walk trial (dual-task). A Wilcoxon signed-rank test showed that there are no significant differences between the single-task and dual-task conditions, except for the ST group (Fig. 2). In the ST group we found that the MAE is significantly higher in dual-task conditions compared to single-task conditions ( $p=0.039, W=5.0)$.

Effect of curved walking and turns. Table 5 shows the mean time errors, MAE and 95\% CI for GE detection during straight-line walking, slalom walking and during turns. A Wilcoxon signed-rank test showed that for all

Table 4 Values for the time errors of the gait events

\begin{tabular}{|c|c|c|c|c|c|c|c|}
\hline & & \multicolumn{6}{|c|}{ Time error (ms) } \\
\hline & & \multicolumn{3}{|c|}{ Initial contacts } & \multicolumn{3}{|c|}{ Final contacts } \\
\hline & & Mean $\pm s d^{1}$ & MAE & $95 \% \mathrm{Cl}$ & $\operatorname{Mean} \pm s d^{1}$ & MAE & $95 \% \mathrm{Cl}$ \\
\hline OA & Straight-line & $14 \pm 36$ & 32 & {$[-57,85]$} & $-25 \pm 33$ & 31 & {$[-90,39]$} \\
\hline OA & Stroop-and-walk & $21 \pm 33$ & 32 & {$[-44,86]$} & $-21 \pm 38$ & 33 & {$[-96,54]$} \\
\hline PD & Straight-line & $26 \pm 29$ & 33 & {$[-30,83]$} & $-25 \pm 45$ & 40 & {$[-113,63]$} \\
\hline PD & Stroop-and-walk & $11 \pm 40$ & 33 & {$[-67,89]$} & $-43 \pm 49$ & 51 & {$[-139,52]$} \\
\hline ST & Straight-line & $17 \pm 36$ & 31 & {$[-54,87]$} & $-6 \pm 33$ & 29 & {$[-71,59]$} \\
\hline ST & Stroop-and-walk & $31 \pm 43$ & 41 & {$[-53,116]$} & $-4 \pm 38$ & 32 & {$[-79,71]$} \\
\hline
\end{tabular}

${ }^{1}$ sd: standard deviation
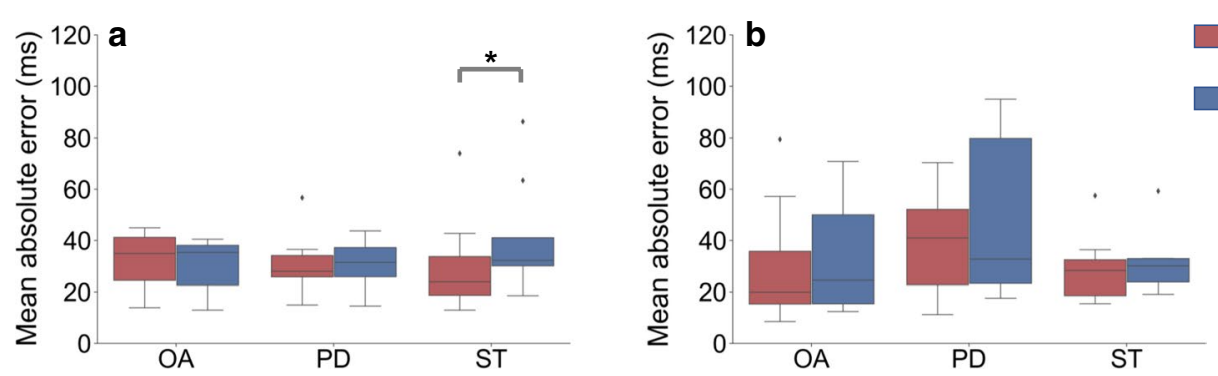

Fig. 2 Mean absolute errors. Boxplots showing the mean absolute errors for the $\mathbf{a}$ initial contacts and $\mathbf{b}$ final contacts detection for the gait events during straight-line walking under single-task (red) or dual-task (blue) conditions. *: $p<0.05$

Table 5 Values for the time errors of the gait events

\begin{tabular}{|c|c|c|c|c|c|c|c|}
\hline & & \multicolumn{6}{|c|}{ Time error (ms) } \\
\hline & & \multicolumn{3}{|c|}{ Initial contacts } & \multicolumn{3}{|c|}{ Final contacts } \\
\hline & & Mean $\pm \mathrm{sd}$ & MAE & $95 \% \mathrm{Cl}$ & Mean \pm sd & MAE & $95 \% \mathrm{Cl}$ \\
\hline OA & Straight-line & $14 \pm 36$ & 32 & {$[-57,85]$} & $-25 \pm 33$ & 31 & {$[-90,39]$} \\
\hline $\mathrm{OA}$ & Slalom & $18 \pm 32$ & 30 & {$[-45,81]$} & $-2 \pm 31$ & 25 & {$[-63,59]$} \\
\hline $\mathrm{OA}$ & Stroop-and-walk (turns) & $11 \pm 57$ & 40 & {$[-101,123]$} & $-33 \pm 77$ & 58 & {$[-183,117]$} \\
\hline PD & Straight-line & $26 \pm 29$ & 33 & {$[-30,83]$} & $-25 \pm 45$ & 40 & {$[-113,63]$} \\
\hline PD & Slalom & $20 \pm 33$ & 30 & {$[-44,83]$} & $-15 \pm 45$ & 35 & {$[-103,72]$} \\
\hline PD & Stroop-and-walk (turns) & $-11 \pm 81$ & 56 & {$[-170,148]$} & $-45 \pm 75$ & 68 & {$[-192,103]$} \\
\hline ST & Straight-line & $17 \pm 36$ & 31 & {$[-54,87]$} & $-6 \pm 33$ & 29 & {$[-71,59]$} \\
\hline ST & Slalom & $19 \pm 36$ & 31 & {$[-53,90]$} & $-4 \pm 34$ & 28 & {$[-72,63]$} \\
\hline ST & Stroop-and-walk (turns) & $12 \pm 62$ & 47 & {$[-110,133]$} & $-7 \pm 77$ & 54 & {$[-158,144]$} \\
\hline
\end{tabular}


groups there are no significant differences between the straight-line walking and the slalom walking, for both ICs and FCs. It is also shown that in IC detection significant differences exist for the MAE of the straigt-line walking and turns, and the slalom walking and turn, for both the PD and ST group (Fig. 3). For the FC detection, significant differences were observed for the MAEs between straight-line walking and turns, and slalom walking and turns, for all groups.

\section{Effect of group on gait event detection}

A Wilcoxon rank-sum test showed that no significant differences were observed between groups for all walking tasks, except for slalom walking, where in FC detection the MAE is significantly larger in the PD group compared to the OA group $(p=0.039, W=-2.608)$.

\section{Discussion}

In this study, shank-mounted IMU-based detection of gait events was tested for different walking tasks and in different mobility-limiting chronic diseases. The detection performance was evaluated for GEs from steps during straight-line walking under both single-task and dual-task conditions. Furthermore, the detection performance was evaluated for GEs from steps during straightline walking, curved walking and turns. Three different groups of participants were distinguished (OA, PD, ST; Table 1) to evaluate whether the detection performance is affected by presence or history of neurological disease.

The high (i.e., almost perfect) recall, precision and F1 score (Table 2) imply that IMU-based detection of ICs and FCs is feasible in both single-task and dual-task straight-line walking for participants with physiological and different pathological walking patterns. Similarly, the high (i.e., again almost perfect) recall, precision and F1 score (Table 3) imply that IMU-based gait event detection is feasible in curved walking by assessing the detection performance in slalom walking. Again, results hold for participants across different walking conditions.
The performance of detecting gait events during turning shows lower recall, precision and F1 score which suggests that shank-mounted IMUs are less feasible to detect GEs during turning. It should be noted that this holds only for how currently the signals were processed. If the vertical acceleration signal would also have been used, like in [37] for lower limb amputees, then GE detection would likely have been less dependent on the (forward) swinging motion of the leg.

Concerning the differences between IMU-based event timings and reference event timings, results were in a range similar to previous studies $[30,35]$. There are many possible contributors to the time difference, and possibly a combination of these will be in play. First, and most importantly, two different systems were used and distinct signal characteristics were used to identify the same event. For the reference system, local extrema in the marker velocity and acceleration marked the instances of the event. [28, 29] For the IMUs, local extrema in the angular velocity about the medio-lateral axis marked the instances of gait events. [17, 18, 32] More recent research found that there was no clear feature from the angular velocity signal related to FC, at least when walking on a treadmill. [38] Next, the filtering of the angular velocity signal may contribute to the time error, as with a lower cut-off frequency less signal details were preserved which affects the presence of local extrema. [39] Potentially, there is also a minor contribution to the time error from the hardware-triggered synchronization [31], as mentioned before ([30, 40, 41]). Most importantly, for all groups and walking tasks, the $95 \% \mathrm{CI}$ of the estimated event timing encompassed $0 \mathrm{~s}$ and therefore the current methods are considered valid for detection of gait events $[17,18,32]$. The $95 \% \mathrm{CI}$ is largest for the time errors of IC and FC during turns.

It was found that the absolute time error is not significantly different when comparing detection of GEs for single-task conditions to dual-task conditions, except for the detection of ICs for the ST group. This may be explained
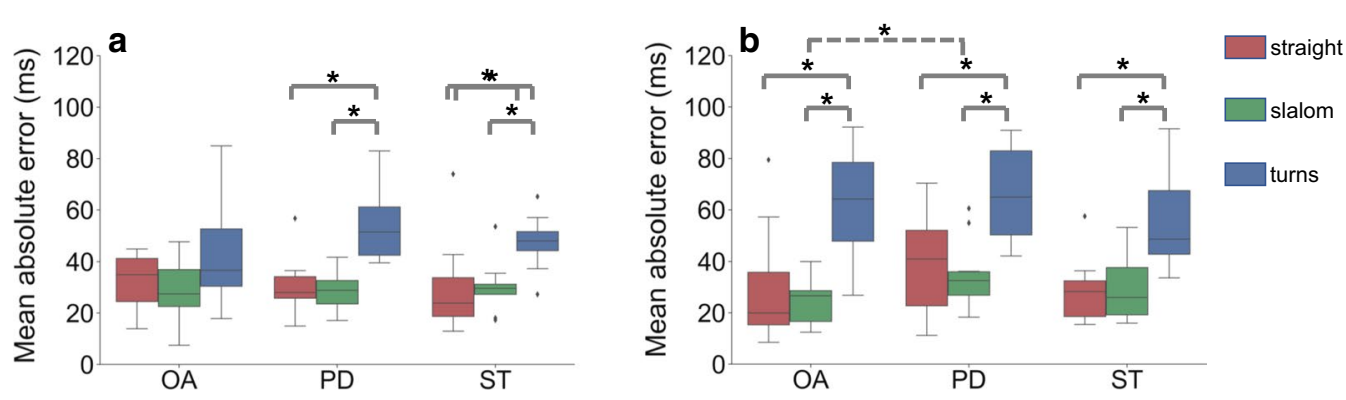

Fig. 3 Mean absolute errors. Boxplots showing the mean absolute errors for the $\mathbf{a}$ initial contacts and $\mathbf{b}$ final contacts detection for the gait events during straight-line walking (red), slalom walking (green) and during turns (blue). *: $p<0.05$ 
by the altered gait pattern that can be observed in some post-stroke patients, especially while executing a cognitive-motor dual-task $[42,43]$. The mean absolute time error was found significantly higher for IC and FC detection during turns compared to both straight-line and slalom walking for the PD and ST group. Together with the lower recall, precision, F1 score and the larger 95\% CI this implies that a shank-mounted IMU is less feasible for detecting GEs during turning. What are the most probable reasons for this observation? In straight-line walking, the leg is swung forward reaching a peak angular velocity at approximately mid-swing $[17,18,32]$. The local maxima right before and after the peak are then correlated to the instances of FC and IC [17, 18, 32]. However, for steps during turning, this swinging motion may not always be observed, depending on which turning strategy is used [44] which may explain the higher number of FN and FP events during turns, compared to straight-line and, especially, slalom walking. The study has in our opinion a particular clinical relevance, considering that increasingly home-derived data will be used for patient management [6] and assessment in clinical trials [45, 46]. IMUs are ideally suited for this performance-based assessment. Our study suggests that the temporal components necessary for the qualitative assessment of gait (IC and FC) can be detected very reliably during straight and slalom walking (e.g. go for a stroll or shopping, commuting to work), but gait phases with rotations of e.g. $180^{\circ}$ and possibly interrupted forward movement can be detected less reliably. This implies that turns should definitely be included in unsupervised IMU-based gait detection. Of course, this statement only applies to the sensor constellation as used in this study, and not, for example, to data from IMUs positioned at the low back. Although methods derived from low back- or foot-mounted IMUs [12, $13,20,47]$ may be less susceptible to turns, literature suggested that GE detection is easier and more robust from shank-mounted IMUs. [17-19]

Furthermore, to continue improving the current methods, and to be less dependent on the forward swinging motion of the leg, future research may also include vertical acceleration signals $[13,37]$ or include information from both the time and frequency domain $[34,48]$.

One of the limitations of this study is the relatively short walking distance. However, the focus of our research was on detecting GEs regardless of the walking distance. Another limitation is that the results are from a supervised assessment in a controlled environment, which is not representative of daily-life conditions $[5,6]$.

\section{Conclusion}

Shank-mounted IMUs can be used to detect gait events from steps during straight-line and curved walking, under both single-task and dual-task conditions, in different neurological populations. Gait events from steps during turns can be detected but result in more missed events and more false events. In case spatio-temporal parameters are subsequently derived, the higher number of missed and false events will have a negative effect on these parameters. If turns are not automatically identified, the spatio-temporal parameters from ambulatory assessment should be interpreted with care.

\section{Abbreviations}

FC: Final contact; FN: False negative; FP: False positive; GE: Gait event; IMU: Inertial measurement unit; IC: Initial contact; MAE: Mean absolute error; MoCA: Montreal Cognitive Assessment; OA: Older adult; OMC: Optical motion capture; PD: Parkinson's disease; sd: Standard deviation; ST: Stroke; TP: True positive.

\section{Acknowledgements}

The authors would like to thank Arash Atrsaei for providing feedback on the submission details.

\section{Authors' contributions}

RR performed the study, developed the algorithms, and analyzed the data. EW, CH and WM designed the study protocol. EW conducted data collections. RR and GUS discussed the algorithms. WM found the financial support. RR, EW, CH, JW, GUS and WM discussed the results and revised the manuscript. All authors read and approved the final manuscript.

\section{Funding}

Open Access funding enabled and organized by Projekt DEAL. This research was funded by Keep Control from the EU's Horizon 2020 research and innovation programme under the Marie Skłodowska-Curie grant agreement number 721577.

\section{Availability of data and materials}

The data that was collected and/or analyzed are not publicly available but are available from the authors on reasonable request.

\section{Ethics approval and consent to participate}

The study was approved by the ethical committee of the medical faculty at the University Hospital Schleswig-Holstein (UKSH), No: D438/18.

\section{Consent for publication}

Not applicable.

\section{Competing interests}

The authors declare that they have no competing interests.

\section{Author details}

${ }_{1}^{1}$ Digital Signal Processing and System Theory, Institute of Electrical and Information Engineering, Kiel University, Kaiserstraße 2, 24143 Kiel, Germany. ${ }^{2}$ Neurogeriatrics, Department of Neurology, University Hospital Schleswig-Holstein, Arnold-Heller-Straße 3, Haus D, 24105 Kiel, Germany.

Received: 7 September 2020 Accepted: 26 January 2021

Published online: 06 February 2021 


\section{References}

1. Croce UD, Cereatti A, Mancini M. Gait parameters estimated using inertial measurement units. In: Müller B, Wolf SI, Brueggemann GP, Deng Z, editors. Handbook of Human Motion. Cham: Springer; 2017. p. 1-21.

2. Buckley C, Alcock L, McArdle R, Rehman RZU, Del Din S, Mazzà C, Yarnall $A J$, Rochester $L$. The role of movement analysis in diagnosing and monitoring neurodegenerative conditions: insights from Gait and Postural Control. Brain Sci. 2019;9(2):34. https://doi.org/10.3390/brainsci9020034.

3. Toro B, Nester C, Farren P. A review of observational gait assessment in clinical practice. Physiother Theory Pract. 2003;19(3):137-49. https://doi. org/10.1080/0959398030796.

4. Iosa M, Picerno P, Paolucci S, Morone G. Wearable inertial sensors for human movement analysis. Exp Rev Med Dev. 2016;13(7):641-59. https:// doi.org/10.1080/17434440.2016.1198694.

5. Hillel I, Gazit E, Nieuwboer A, Avanzino L, Rochester L, Cereatti A, Croce UD, Rikkert MO, Bloem BR, Pelosin E, Del Din S, Ginis P, Giladi N, Mirelman A, Hausdorff JM. Is every-day walking in older adults more analogous to dual-task walking or to usual walking? Elucidating the gaps between gait performance in the lab and during 24/7 monitoring. Eur Rev Aging Phys Act. 2019;16(1):6. https://doi.org/10.1186/s11556-019-0214-5.

6. Warmerdam E, Hausdorff JM, Atrsaei A, Zhou Y, Mirelman A, Aminian K, Espay AJ, Hansen C, Evers LJW, Keller A, Lamoth C, Pilotto A, Rochester L, Schmidt G, Bloem BR, Maetzler W. Long-term unsupervised mobility assessment in movement disorders. Lancet Neurol. 2020;19(5):462-70. https://doi.org/10.1016/S1474-4422(19)30397-

7. Haji Ghassemi N, Hannink J, Martindale C, Gaßner H, Müller M, Klucken J, Eskofier B. Segmentation of Gait sequences in sensor-based movement analysis: a comparison of methods in Parkinson's Disease. Sensors. 2018;18(2):145. https://doi.org/10.3390/s18010145.

8. Rueterbories J, Spaich EG, Larsen B, Andersen OK. Methods for gait event detection and analysis in ambulatory systems. Med Eng Phys. 2010;32(6):545-52. https://doi.org/10.1016/j.medengphy.2010.03.007.

9. Richards J, Levine D, Whittle M. Whittle's Gait analysis. Edinburgh: Churchill Livingstone Elsevier; 2012.

10. Perry J, Burnfield J. Gait analysis: normal and pathological function. New Jersey: SLACK Incorporated; 2010.

11. Paraschiv-lonescu A, Newman CJ, Carcreff L, Gerber CN, Armand S, Aminian K. Locomotion and cadence detection using a single trunk-fixed accelerometer: validity for children with cerebral palsy in daily life-like conditions. J NeuroEng Rehabil. 2019;16(1):24. https://doi.org/10.1186/ s12984-019-0494-

12. Pham $M H$, Elshehabi M, Haertner $L$, Del Din $S$, Srulijes $K$, Heger $T$, Synofzik M, Hobert MA, Faber GS, Hansen C, Salkovic D, Ferreira JJ, Berg D, Sanchez-Ferro Á, van Dieën JH, Becker C, Rochester L, Schmidt G, Maetzler W. Validation of a step detection algorithm during straight walking and turning in patients with Parkinson's disease and older adults using an inertial measurement unit at the lower back. Front Neurol. 2017. https:// doi.org/10.3389/fneur.2017.00457

13. McCamley J, Donati M, Grimpampi E, Mazzà C. An enhanced estimate of initial contact and final contact instants of time using lower trunk inertial sensor data. Gait Posture. 2012;36(2):316-8. https://doi.org/10.1016/j. gaitpost.2012.02.019.

14. Zijlstra W, Hof AL. Assessment of spatio-temporal gait parameters from trunk accelerations during human walking. Gait Posture. 2003;18(2):1-10. https://doi.org/10.1016/S0966-6362(02)00190-X.

15. Zijlstra W. Assessment of spatio-temporal parameters during unconstrained walking. Eur J Appl Physiol. 2004;92(1-2):39-44. https://doi. org/10.1007/s00421-004-1041-5.

16. Trojaniello D, Cereatti A, Della Croce U. Accuracy, sensitivity and robustness of five different methods for the estimation of gait temporal parameters using a single inertial sensor mounted on the lower trunk. Gait Posture. 2014;40(4):487-92. https://doi.org/10.1016/j.gaitpost.2014.07.007.

17. Salarian A, Russmann H, Vingerhoets FJG, Dehollain C, Blanc Y, Burkhard PR, Aminian K. Gait Assessment in Parkinson's Disease: toward an ambulatory system for long-term monitoring. IEEE Trans Biomed Eng. 2004;51(8):1434-43. https://doi.org/10.1109/TBME.2004.827933.

18. Trojaniello D, Cereatti A, Pelosin E, Avanzino L, Mirelman A, Hausdorff JM, Della Croce U. Estimation of step-by-step spatio-temporal parameters of normal and impaired gait using shank-mounted magnetoinertial sensors: application to elderly, hemiparetic, parkinsonian and choreic gait. J NeuroEng Rehabil. 2014;11(1):152. https://doi. org/10.1186/1743-0003-11-152.

19. Kong W, Lin J, Waaning L, Sessa S, Cosentino S, Magistro D, Zecca M Kawashima R, Takanishi A Comparison of gait event detection from shanks and feet in single-task and multi-task walking of healthy older adults. In: IEEE (ed.) 2016 IEEE International Conference on Robotics and Biomimetics (ROBIO), 2016;pp. 2063-2068. https://doi.org/10.1109/ROBIO .2016 .7866633

20. Laudanski A, Yang Shuozhi, Li Qingguo A concurrent comparison of inertia sensor-based walking speed estimation methods. In: IEEE (ed.) 2011 Annual International Conference of the IEEE Engineering in Medicine and Biology Society, 2011;pp. 3484-3487. https://doi.org/10.1109/IEMBS .2011 .6090941

21. Del Din S, Godfrey A, Rochester L. Validation of an accelerometer to quantify a comprehensive battery of gait characteristics in healthy older adults and Parkinson's disease: toward clinical and at home use. IEEE J Biomed Health Inform. 2016;20(3):838-47. https://doi.org/10.1109/ JBHI.2015.2419317.

22. Glaister BC, Bernatz GC, Klute GK, Orendurff MS. Video task analysis of turning during activities of daily living. Gait Posture. 2007;25(2):289-94. https://doi.org/10.1016/j.gaitpost.2006.04.003.

23. Turcato AM, Godi M, Giardini M, Arcolin I, Nardone A, Giordano A, Schieppati M. Abnormal gait pattern emerges during curved trajectories in high-functioning Parkinsonian patients walking in line at normal speed. PLoS ONE. 2018;13(5):0197264. https://doi.org/10.1371/journ al.pone.0197264.

24. Dijkstra B, Kamsma YP, Zijlstra W. Detection of Gait and postures using a miniaturized triaxial accelerometer-based system: accuracy in patients with mild to moderate Parkinson's disease. Arch Phys Med Rehabi. 2010;91(8):1272-7. https://doi.org/10.1016/j.apmr.2010.05.004.

25. Nasreddine ZS, Phillips NA, Bédirian V, Charbonneau S, Whitehead V, Collin I, Cummings JL, Chertkow H. The Montreal Cognitive Assessment, MoCA: A Brief Screening Tool For Mild Cognitive Impairment. Journal of the American Geriatrics Society. 2005;53(4):695-9. https://doi. org/10.1080/09593980307969.

26. Henik A, Tzelgov J. Is three greater than five: the relation between physical and semantic size in comparison tasks. Mem Cogn. 1982;10(4):389-95. https://doi.org/10.3758/BF03202431.

27. Gløersen $\varnothing$, Federolf P. Predicting missing marker trajectories in human motion data using marker intercorrelations. PLOS ONE. 2016;11(3):0152616. https://doi.org/10.1371/journal.pone.0152616.

28. Hreljac A, Marshall RN. Algorithms to determine event timing during normal walking using kinematic data. J Biomech. 2000;33(6):783-6. https ://doi.org/10.1016/S0021-9290(00)00014-2.

29. Pijnappels M, Bobbert MF, van Dieën JH. Changes in walking pattern caused by the possibility of a tripping reaction. Gait Posture. 2001;14(1):11-8. https://doi.org/10.1016/S0966-6362(01)00110-2.

30. Carcreff L, Gerber C, Paraschiv-lonescu A, De Coulon G, Newman C, Armand S, Aminian K. What is the best configuration of wearable sensors to measure spatiotemporal gait parameters in children with cerebral palsy? Sensors. 2018;18(2):394. https://doi.org/10.3390/s18020394.

31. Qualisys AB: Qualisys Track Manager: User Manual. 2011

32. Aminian K, Najafi B, Büla C, Leyvraz P-F, Robert P. Spatio-temporal parameters of gait measured by an ambulatory system using miniature gyroscopes. J Biomech. 2002;35(5):689-99. https://doi.org/10.1016/S0021 -9290(02)00008-8.

33. Najafi B, Aminian K, Paraschiv-lonescu A, Loew F, Bula CJ, Robert P. Ambulatory system for human motion analysis using a kinematic sensor: monitoring of daily physical activity in the elderly. IEEE Trans Biomed Eng. 2003;50(6):711-23. https://doi.org/10.1109/TBME.2003.812189.

34. Khandelwal S, Wickstrom N. Gait event detection in real-world environment for long-term applications: incorporating domain knowledge into time-frequency analysis. IEEE Trans Neural Syst Rehabil Eng. 2016:24(12):1363-72. https://doi.org/10.1109/TNSRE.2016.2536278.

35. Ji N, Zhou H, Guo K, Samuel O, Huang Z, Xu L, Li G. Appropriate mother wavelets for continuous gait event detection based on time-frequency analysis for hemiplegic and healthy individuals. Sensors. 2019;19(16):3462. https://doi.org/10.3390/s19163462.

36. Wilcoxon F. Individual comparisons by Ranking methods. Biometr Bull. 1945;1(6):80. https://doi.org/10.2307/3001968. 
37. Maqbool HF, Husman MAB, Awad MI, Abouhossein A, Mehryar P, lqbal N, Dehghani-Sanij AA Real-time gait event detection for lower limb amputees using a single wearable sensor. In: 2016 38th Annual International Conference of the IEEE Engineering in Medicine and Biology Society (EMBC), 2016;pp. 5067-5070. https://doi.org/10.1109/EMBC.2016.75918 66

38. Bötzel K, Marti FM, Ángel Carvajal Rodríguez M, Plate A, Vicente AO. Gait recording with inertial sensors - How to determine initial and terminal contact. J Biomech. 2016;49(3):332-7. https://doi.org/10.1016/j.jbiom ech.2015.12.035.

39. Catalfamo P, Ghoussayni S, Ewins D. Gait event detection on level ground and incline walking using a rate gyroscope. Sensors. 2010;10(6):5683-702. https://doi.org/10.3390/s100605683.

40. Stanhope SJ, Kepple TM, McGuire DA, Roman NL. Kinematic-based technique for event time determination during gait. Med Biol Eng Comput. 1990;28(4):355-60. https://doi.org/10.1007/BF02446154.

41. Chiari L, Croce UD, Leardini A, Cappozzo A. Human movement analysis using stereophotogrammetry. Gait Posture. 2005;21(2):197-21111. https ://doi.org/10.1016/j.gaitpost.2004.04.004.

42. Baetens T, De Kegel A, Palmans T, Oostra K, Vanderstraeten G, Cambier D. Gait analysis with cognitive-motor dual tasks to distinguish fallers from nonfallers among rehabilitating stroke patients. Arch Phys Med Rehabil. 2013;94(4):680-6. https://doi.org/10.1016/j.apmr.2012.11.023.

43. Timmermans C, Roerdink M, Janssen TWJ, Meskers CGM, Beek PJ. Dual-task walking in challenging environments in people with stroke: cognitive-motor interference and task prioritizatio. Stroke Res Treatm. 2018;2018:1-8. https://doi.org/10.1155/2018/7928597.

44. Hase K, Stein RB. Turning strategies during human walking. J Neurophysiol. 1999;81(6):2914-22. https://doi.org/10.1152/jn.1999.81.6.2914.

45. Cerreta F EMA experience on mHealth technology. Last accessed: 31 August 2020 2018. https://doi.org/10.1080/17434440.2016.11986947

46. Clinical Trials Transformation Institute: CTTI recommendations: developing novel endpoints generated by mobile technology for use in clinical trials. Last accessed: 31 August 2020 2017. https://doi.org/10.1080/17434 440.2016.11986948

47. Rampp A, Barth J, Schülein S, Gaßmann K, Klucken J, Eskofier BM. Inertial sensor-based stride parameter calculation from gait sequences in geriatric patients. IEEE Trans Biomed Eng. 2015;62(4):1089-97. https://doi. org/10.1109/TBME.2014.2368211.

48. Cain SM, Porter VM, Ojeda L, Perkines NC Accurate and robust gait event detection using foot-mounted inertial measurement units. In: $41 \mathrm{st}$ Annual Meeting of the American Society of Biomechanics 2017.

\section{Publisher's Note}

Springer Nature remains neutral with regard to jurisdictional claims in published maps and institutional affiliations.
Ready to submit your research? Choose BMC and benefit from:

- fast, convenient online submission

- thorough peer review by experienced researchers in your field

- rapid publication on acceptance

- support for research data, including large and complex data types

- gold Open Access which fosters wider collaboration and increased citations

- maximum visibility for your research: over 100M website views per year

At BMC, research is always in progress.

Learn more biomedcentral.com/submissions 